\title{
Grounded theory : quels usages dans les recherches en contrôle de gestion ?*
}

\author{
Vassili JOANNIDES ** \\ ESC La Rochelle \\ vassili@gmx.ch \\ Nicolas BERLAND \\ Université Paris Dauphine, DRM - CREFIGE \\ nicolas.berland@dauphine.fr
}

\section{Résumé :}

Cet article a pour objet de présenter les principaux enjeux liés à la production de connaissances avec la grounded theory en contrôle de gestion. La recherche est une étude bibliographique confrontant les pratiques de la grounded theory avec les recommandations de ses fondateurs (Glaser et Strauss, 1967; Strauss et Corbin, 1998). Après avoir présenté les principes et usages de la grounded theory, nous montrons la diversité d'utilisation de cette méthodologie dans les recherches en contrôle de gestion à travers onze articles revendiquant ce positionnement.

Mots-clés: grounded theory, connaissance, recherche qualitative, conceptualisation, théorisation

Grounded theory: what uses in management accounting research?

\begin{abstract}
:
The present paper draws on the main issues in the production of scientific knowledge based upon grounded theory in management accounting research. This piece of work is a bibliographic study confronting grounded theory practices with the expectations of its founders (Glaser et Strauss, 1967; Strauss et Corbin, 1998). After we shed light on the principles and issues in the discovery of a grounded theory, we bring insights into the variety of uses thereof throughout eleven articles claiming that positioning.
\end{abstract}

Keywords: grounded theory, knowledge, qualitative research, conceptualising, theorising

* Publié avec le concours de la FNEGE et de la Commission Européenne (programme Marie Curie)

** Auteur à contacter.

\section{Remerciements :}

Nous remercions notre réviseur anonyme pour ses remarques et ses commentaires. Nous tenons aussi à exprimer notre gratitude aux doctorants de Nicolas Berland qui se sont souvent montrés encore plus sévères que notre réviseur. Nous sommes également très reconnaissants à Sven Modell et Deryl Northcott pour leurs conseils et remarques tout au long de ce travail. 


\section{INTRODUCTION}

La grounded theory est un processus de production de connaissances en sciences sociales développé par Glaser et Strauss à partir de 1967. Cette approche de recherche abductive propose d'élaborer des théories directement à partir des donnés empiriques renversant la relation traditionnelle entre théories, hypothèses et données et faisant une plus large place à l'observation. Alors que de nombreuses branches des sciences sociales (sociologie, anthropologie, science politique) se sont approprié peu à peu la grounded theory, elle reste encore peu utilisée en recherche comptable tant en France que dans le monde anglo-saxon. Un certain nombre d'idées reçues et d'interprétations erronées sur la nature de ce processus de recherche l'ont pour le moment laissé en marge des enseignements en méthodologie de la recherche. Son présupposé est en effet audacieux, sinon dérangeant, pour le chercheur. Il consiste à faire abstraction des cadres théoriques préexistants afin de laisser un objet d'étude produire de lui-même des éléments constitutifs d'une théorie. Alors que les canons traditionnels de la recherche ont des visées explicatives, la grounded theory compte au rang de ses objectifs la compréhension d'un objet et par extension d'un environnement social ou managérial (Quattrone, 2000).

L'une des hypothèses de cette approche est que les études antérieures n'ont pas vu ou ont négligé des dimensions empiriques qui font défaut à la progression de la connaissance (Becker, 2001). L'examen minutieux du terrain conduit le chercheur à découvrir une nouvelle théorie ou à recourir à la théorie la plus appropriée parmi celles déjà existantes pour comprendre son objet. Le développement d'une théorie enracinée dans un terrain d'étude est un mode de recherche alternatif au modèle hypothético-déductif dominant. Ceci est une approche résolument qualitative fondée sur des démarches abductives (Glaser et Strauss, 1967, Strauss et Corbin, 1990, 1998, David, 2000) 
Nous proposons d'étudier comment et à quelles conditions ce processus de recherche peut produire de la connaissance utile aux chercheurs. Dans cette perspective, l'article est découpé en deux parties. La première présente les enjeux de la grounded theory pour la production de connaissances. La deuxième partie examine les utilisations concrètes et les apports de ce type de recherche en contrôle de gestion.

\section{LA GROUNDED THEORY, UN MODE ALTERNATIF DE PRODUCTION DE CONNAISSANCES}

Considérant la grounded theory comme un mode de recherche alternatif au canon hypothéticodéductif et comme une modalité dans un cadre interprétatif de type abductif, nous présentons dans cette première partie ses spécificités. Nous insisterons dans un premier temps sur la production de connaissances qu'elle permet. Dans un second temps, nous présenterons ses critères de validité trop souvent ignorés ou méconnus.

\subsection{Produire de la connaissance avec la grounded theory}

La grounded theory permet de produire trois types de connaissance: théorique, empirique ou méthodologique (Quattrone, 2000). Cette richesse supposée de connaissances productibles par la grounded theory est enracinée dans des contextes scientifiques spécifiques.

\subsubsection{Trois types de connaissances}

La grounded theory peut produire trois types de connaissances : la création ou l'enrichissement de théories, une meilleure connaissance empirique des objets de recherche, de nouvelles 
méthodologies.

\subsubsection{Générer de nouvelles théories ou en enrichir d'anciennes}

L'objectif principal de la grounded theory est, à partir d'une observation empirique, de découvrir et de formuler une théorie que Glaser \& Strauss (1967) qualifient selon les cas de formelle, de substantive ou de confirmatoire. Une théorie formelle apparaît comme une théorie achevée dont les concepts sont susceptibles d'être exploités dans d'autres travaux. Par comparaison, une théorie substantive est moins achevée. Elle pose uniquement des concepts qui pourront servir au développement d'une théorie formelle dans des travaux ultérieurs. Une théorie confirmatoire vient confirmer ou enrichir une théorie déjà existante. L'absence de préjugés théoriques permet une compréhension d'abord naïve du terrain. Soit une théorie nouvelle est produite à partir du phénomène observé, soit les concepts émergeant de l'observation empirique se rapportent directement à une théorie déjà existante. Il revient alors au chercheur de l'enrichir à l'aune de ses observations.

La construction de connaissances vient de ce que le chercheur est amené à d'abord déconstruire son objet d'étude avant de le reconstruire au regard de ce qu'il en a compris. La découverte d'une théorie constitue le stade final de la recherche et guide ensuite le processus d'écriture. Tout d'abord, la conduite d'une étude exploratoire fait émerger des concepts et une théorie provisoires. Au fur et à mesure que le chercheur reconstitue les connexions entre les différentes composantes du terrain, sa théorie évolue. La conceptualisation de ces relations constitue le cadre théorique final de la recherche. Par un processus récursif, l'application de ce cadre permet de restituer le cas pour le lecteur. Celui-ci n'est plus une simple histoire mais une suite de données empiriques reliées par des concepts issus des observations de terrain. La restitution est donc très exigeante en termes de 
rigueur d'écriture comme dans n'importe quelle autre recherche interprétativiste / constructiviste (Thiétart, 1999, Quattrone, 2000, Charreire et Huault, 2002)

1.1.1.2. Détailler les études empiriques pour mieux connaître les objets de recherche

Glaser et Strauss précisent que la description du terrain de recherche, fondée sur des concepts clairs, porte en elle-même les éléments permettant l'émergence d'une théorie. Pour pouvoir ainsi produire des connaissances empiriques, il est nécessaire que la description soit la plus exhaustive et précise possible.

Selon Locke (2000), il est tout aussi important de disposer de connaissances empiriques sur un objet que de cadres théoriques. Le décrire à l'aide de concepts permet de publier des études de cas qui pourront alors être réexploitées dans d'autres recherches ou à des fins pédagogiques (Howard et Stout, 2006). Les cas publiés peuvent porter à la connaissance du public des modes de contrôle encore inconnus et ainsi déclencher de futures recherches (Booth, 1993, Laughlin, 1995).

\subsubsection{Favoriser l'assemblage et l'innovation méthodologiques}

Glaser et Strauss préconisent la combinaison de d'ethnométhodologies allant de l'observation passive à l'observation participante avec des procédés narratifs proches de ceux du romancier ou du nouvelliste, voire du dramaturge. En fait, dans la mesure où il s'agit de produire de meilleures données empiriques ou de faire émerger de nouveaux types de relations, toute innovation dans l'observation et le compte-rendu du terrain doit être favorisée. Strauss et Corbin (1998) mettent toutefois assez classiquement l'accent sur la conduite d'entretiens et l'analyse de discours ou de contenu. Ils insistent sur l'importance des données secondaires alors négligées par Glaser et Strauss. 
Pour les sciences de gestion, Locke (2000) envisage l'étude de cas approfondie en complément d'autres méthodes.

Strauss et Corbin (1998) encouragent le chercheur à combiner les différentes méthodes qui s'offrent à lui, sans rejeter de manière systématique les méthodes d'analyse quantitatives. Compte tenu de la grande variété de combinaisons possibles de méthodologies à la disposition du chercheur, Locke (2000) considère que la pratique de la grounded theory peut mettre au jour des modes de recherche peu usités. Quelle qu'en soit la modalité, la combinaison doit dans tous les cas être guidée par l'objet d'étude et la question de recherche. Systématiquement, les chercheurs recourant à la grounded theory sont dans l'obligation de revenir en conclusion de leur article sur la méthodologie adoptée afin d'en présenter a posteriori l'intérêt. Strauss et Corbin estiment que c'est à cette condition qu'une recherche peut prétendre produire de la connaissance méthodologique.

\subsubsection{Contextes de production de connaissances}

Strauss et Corbin distinguent deux cas de figure dans lesquels il peut être opportun ou nécessaire de découvrir une théorie enracinée dans le terrain. Cette méthode peut «être utilisée pour explorer des domaines sur lesquels on sait peu de choses ou bien des domaines sur lesquels on gagnerait à trouver de nouveaux éléments de compréhension » (Strauss et Corbin, 1998, 11).

Moins intuitive, et pourtant tout aussi intéressante, est l'idée que la grounded theory peut trouver sa place en cas d'objets de recherche déjà très documentés. Strauss et Corbin rappellent qu'il existe parfois un ou plusieurs cadres théoriques pour un même objet. S'il n'en existe qu'un, le chercheur, en découvrant une autre compréhension théorique, permettra un travail de comparaison avec la théorie préexistante. Celle-ci ne pourra être tenue pour acquise que lorsque plusieurs travaux 
aboutiront à des conclusions compatibles. Au final, c'est la collection des cadres utilisés et découverts qui permet une compréhension idéale de l'objet.

Lorsqu'un domaine a déjà fait l'objet de nombreuses études et jouit de compréhensions multiples, Strauss et Corbin considèrent que la grounded theory peut apporter des perspectives nouvelles. En reprenant le processus de théorisation depuis le commencement, le chercheur appréhende le terrain

comme un objet nouveau. Plus que jamais, il est indispensable qu'il fasse fi de tout cadre conceptuel connu sur cet objet. La nouvelle compréhension ainsi avérée peut se surajouter aux précédentes et finalement redonner à un objet d'étude considéré comme épuisé un intérêt scientifique.

\subsection{Les critères de validité de la démarche}

Strauss et Corbin proposent trois critères pour apprécier la validi té de la recherche : le codage des données, le développement d'une attitude réflexive par le chercheur et la plausibilité de la narration. Les deux derniers empruntent explicitement au constructivisme (Thiétart, 1999, Quattrone, 2000, Charreire et Huault, 2002).

\subsubsection{Le codage des données textuelles}

En quête de légitimité scientifique, la grounded theory a adapté les outils de la recherche qualitative et développé les siens. Selon Strauss et Corbin (1998), les données issues du terrain se trouvent dans le discours des individus. Celui-ci doit alors être soigneusement enregistré et retranscrit. Il peut ensuite faire l'objet d'un codage ouvert (open coding) par le chercheur. Cette technique doit permettre d'identifier des catégories afin de classer des pans du discours des individus par mots- 
clés. Le codage doit alors faire l'objet de plusieurs itérations au gré des contacts avec le terrain et la littérature existante dans laquelle Strauss et Corbin (1998) voient des donnés secondaires susceptibles d'aider à la compréhension de l'objet étudié. Le codage se fait de manière abductive par itérations successives et regroupements progressifs. Si les catégories obtenues sont trop étonnantes par rapport à celles déjà existantes, le chercheur doit être capable de comprendre l'origine de ces divergences. Si dans la littérature, des classifications satisfaisantes pour l'objet étudié existent, le chercheur pourra s'y référer et les emprunter. Mais si aucune typologie préexistante ne lui paraît suffisamment explicative, il lui est loisible d'en proposer de nouvelles qui seront directement issues de son terrain.

Dans un second temps vient l'analyse des résultats du codage ouvert. Celle-ci consiste à retrouver des similitudes et des relations dans les différentes formes de discours (axial coding) à l'aune des catégories identifiées dans l'étape précédente. Finalement, le chercheur doit sélectionner le phénomène central qui ressort du codage axial et qui constitue la structure émergente de sa théorie (selective coding). Tout comme pour la classification, il est invité à comparer ses résultats ex post aux travaux antérieurs et à proposer les déclinaisons propres à son terrain. Celles-ci deviendront une théorie à part entière. S'il n'existe pas littérature, il est de sa responsabilité d'appeler des travaux ultérieurs à évaluer la théorie qu'il a ainsi découverte.

\subsubsection{La réflexivité des acteurs vis-à-vis du texte}

Glaser et Strauss proposent un autre critère de validité pour évaluer les conclusions auxquelles conduit la grounded theory, tenant et à la possibilité de narrer l'observation et à la transmissibilité des connaissances. Les acteurs sous observation doivent être en mesure de comprendre et d'accepter le texte qu'a produit le chercheur. Il convient qu'à sa lecture, ils puissent reconnaître l'organisation 
ou se reconnaître eux-mêmes. On peut ainsi rapprocher ce critère de narrativité à celui d'enseignabilité des travaux constructivistes (Quattrone, 2000, Charreire et Huault, 2002). La recherche doit impliquer tous les acteurs de manière réflexive. Idéalement, dans l'application de la grounded theory, il conviendrait que le chercheur soumette systématiquement ses conclusions aux membres du groupe qu'il a observés ou rencontrés ainsi qu'à des personnes de l'organisation qu'il ne connaît pas pour validation. Pour être en mesure de prouver la scientificité de son travail, il doit également produire ces remarques et commentaires dans l'article.

Glaser et Strauss précisent que le texte doit permettre au lecteur, membre du groupe étudié, d'agir. Il s'agit moins de formuler des recommandations d'ordre normatif que d'amener le lecteur à réfléchir à ses propres actions, à leurs motivations, à leur contenu ainsi qu'à leurs effets. Les individus doivent alors être en mesure de donner du sens à leurs actions passées et présentes ou penser leurs actions à venir en fonction des observations qu'a pu faire le chercheur. Pour Locke, la retombée managériale de la recherche viendra directement de cette capacité réflexive. Glaser et Strauss envisagent ainsi que le chercheur retourne vers son objet d'étude une fois la recherche terminée pour mesurer son impact sur les décisions ou actions. Il devra introduire les commentaires postopératoires des acteurs organisationnels dans son rapport de recherche afin de prouver sa validité scientifique.

\subsubsection{La plausibilité du discours}

Le respect des critères de scientificité proposés par Glaser et Strauss rend la recherche fondée sur la grounded theory tributaire des délais de lecture et de la capacité réflexive des acteurs. Les deux critères précédents peuvent apparaître comme un idéal contraignant. Pour cette raison, Parker et Roffey (1997) estiment que des critères plus souples peuvent permettre de juger de la scientificité 
d'une recherche fondée sur la grounded theory. Selon eux, la plausibilité du discours peut suffire. Pourra alors être validée une recherche dont les descriptions et les enchaînements conceptuels sont convaincants.

A défaut de capacité réflexive des acteurs, la recherche doit permettre d'identifier les individus ainsi que les lieux et les mécanismes présentés. Le lecteur extérieur doit être en mesure de s'imaginer le fonctionnement de l'organisation étudiée et de s'en représenter les acteurs. La réflexivité du texte est in fine orientée vers le lecteur externe. Locke (2000) voit la qualité d'écriture comme un élément de scientificité, puisque c'est elle qui convainc ou non le lecteur de la réalité rapportée. La validité de la recherche est aussi donnée par les réviseurs nommés par les éditeurs des revues (Howard et Stout, 2006) en tant que lecteurs externes avertis et compétents.

La présentation de ces enjeux liés à l'adoption d'une démarche de grounded theory révèlent que sa pratique ne peut être monolithique. Les modalités d'un tel positionnement offrent des voies plurielles vers la production de connaissances.

\section{LA GROUNDED THEORY : DES VOIES PLURIELLES VERS LA PRODUCTION DE CONNAISSANCES}

Cette deuxième partie est divisée en cinq sections. La première présente tout d'abord la méthodologie employée pour apprécier les usages de la grounded theory dans les recherches en contrôle de gestion. Les quatre suivantes développeront les résultats de l'analyse des travaux sélectionnés. La deuxième section étudiera les questions de recherche. La troisième détaillera les modes opératoires de la grounded theory. La quatrième s'intéressera aux apports effectifs de la grounded theory. Enfin, la cinquième section sera consacrée à l'examen des critères de scientificité 
retenus par les auteurs.

\subsection{Méthodologie et données : une étude bibliographique multi-critères}

Pour comprendre comment les chercheurs en contrôle de gestion utilisent la grounded theory, nous avons procédé à une étude bibliographique. Notre objectif n'est pas de présenter toutes les utilisations de la grounded theory, mais de rendre intelligible la diversité des voies de recherche en offrant un éclairage sur certains modes opératoires.

Pour les publications en langue anglaise, nous avons collecté nos données dans les bases de données des principaux éditeurs internationaux. Nous avons retenu des articles utilisant la démarche de la grounded theory en contrôle de gestion. Nous avons laissé de côté les travaux uniquement épistémologiques ou méthodologiques. Le premier critère de sélection était le champ de recherche « comptabilité ». Pour cela, nous avons isolé toutes les revues dont le titre contenait le terme «accounting ». Celles-ci publiant des travaux aussi bien en comptabilité qu'en contrôle de gestion ou audit, nous avons croisé ce critère avec une recherche par thème. Seuls devaient être sélectionnés des articles dont le thème contenait les termes «management accounting 》 ou «management control».

Nous avons ensuite procédé par itérations successives. Dans un premier temps, nous avons retenu les articles dans le corps du texte desquels apparaissait l'expression « grounded theory ». Certains auteurs se référant aux fondateurs de la démarche sans nécessairement la nommer, nous avons ensuite cherché les articles dont le corps de texte contenait les noms propres « Glaser», «Strauss », ou «Corbin ». Afin de ne pas négliger les articles dans lesquels l'expression «grounded theory» ou les noms propres ne sont référencés qu'en bibliographie, nous avons cherché à identifier ces 
références implicites en appliquant les mêmes mots-clés aux références bibliographiques. Nous avons pu identifier onze articles remplissant ces critères dans les revues anglo-saxonnes.

Nous avons appliqué la même démarche aux revues francophones. Dans la revue Comptabilité Contrôle Audit (CCA), analysée de sa création à début 2008, quatre articles font mention en bibliographie des références de Glaser et Strass ou Strauss et Corbin, ou seulement de l'un de ces trois auteurs. Un article traite de comptabilité financière (Durocher, et al., 2004), un autre d'audit (Richard et Reix, 2002), tandis qu'un n'utilise la grouded theory que de façon très secondaire pour construire un questionnaire administré par voie postale qui occupe ensuite le cœur de l'article (Villesèque-Dubus, 2005). Enfin, un auteur cite Corbin en bibliographie sans utiliser de méthodologie en lien direct avec la grounded theory (Nobre, 2001). Ces articles se trouvent donc hors de notre champ. Nous avons également analysé le contenu de Finance, Contrôle, Stratégie depuis sa création en 1998. Nous n’y avons trouvé aucun article en contrôle de gestion utilisant la grounded theory. En revanche, des thèses de doctorat la mobilisent (Chambost, 2007). Par souci de cohérence avec le traitement des sources en langue anglaise, nous n'avons pas retenu ces références.

Cette recension confirme le point de vue souvent exprimé par les auteurs de notre échantillon que les articles de recherche utilisant la grounded theory sont finalement assez rares. Le détail de notre échantillon est fourni en annexe 1.

Nous avons reformulé chaque article selon une grille d'analyse systématique (annexes 1 et 2) afin d'identifier aussi bien la diversité d'utilisations de la grounded theory que les invariants d'une recherche à l'autre. Cette grille se compose de quatre catégories correspondant aux enjeux liés à l'utilisation de la grounded theory présentés dans la première partie. Nous les avons nommées : « questions de recherche », «méthodologies», « apports» et «scientificité ». Nous les avons 
ensuite sub-divisées en sous-catégories. La catégorie «questions de recherche » est sub-divisée en «expression de la question de recherche» et en «nouveauté ou préexistence de la question recherche ». La catégorie «apports» est également sub-divisée en trois sous-catégories, «théorique », «empirique » et «méthodologique ». A la lumière des références étudiées, la catégorie «mode d'utilisation» a vu émerger deux sous-catégories, «entretiens » et « ethnométhodologie». Les quatre sections suivantes analysent successivement ces quatre catégories et leurs sub-divisions.

\subsection{Les questions de recherche traitées et le positionnement par rapport à la littérature existante}

Notre échantillon montre que les chercheurs recourent à la grounded theory aussi bien lorsqu'il existe peu ou pas d'études antérieures que lorsque celles-ci sont abondantes. Néanmoins, le choix de la grounded theory motivé par l'absence de travaux représente plus de la moitié de notre échantillon. Dans la plupart des autres cas, c'est le souhait de porter un regard différent sur un thème déjà documenté qui justifie son utilisation.

2.2.1. Des questions de recherche nouvelles et d'autres faussement nouvelles

Sept articles présentent une question de recherche complètement nouvelle ou faussement novatrice. Cinq d'entre eux traitent d'une question offrant de nouvelles voies d'exploration pour la recherche en contrôle. Nous avons ainsi considéré comme novatrice l'étude des facteurs influençant l'utilisation des techniques du contrôle de gestion dans les églises et congrégations religieuses (Parker, 2001, 2002) ou dans les ONG(Goddard et Assad, 2006). Si l'étude des organisations religieuses et des ONG n'est pas nouvelle, les problématiques qui y sont étudiées le sont en revanche pleinement. C'est aussi le cas pour l'étude du lien imprévisible entre modes de contrôle de 
gestion et facteurs culturels, économiques et politiques dans les pays en développement (Wickramasinghe, et al., 2004). De la même manière, la prise en compte des annonces privées éthiques, sociales et environnementales dans l'investissement socialement responsable (Solomon et Solomon, 2006), question centrale de la recherche en finance sur les facteurs de l'investissement responsable, n'a pas encore été étudiée dans les recherches en contrôle. Tous les auteurs insistent sur le fait que le caractère novateur de leur recherche constitue en soi une contribution à la connaissance scientifique.

Dans deux autres articles, les auteurs affirment que le caractère récent de l'intérêt porté à la question traitée confère à celle-ci un caractère novateur. Mais il nous semble que l'étude des rapports entre les managers financiers et les défenseurs de la mission dans une organisation religieuse (Lightbody, 2000) présente un caractère faussement novateur. En effet, cette question, formulée par Laughlin (1988) et Booth (1993), est devenue centrale dans les recherches sur la comptabilité des organisations religieuses (McPhail, et al., 2004, 2005). Dans le même ordre d'idées, l'étude des facteurs influençant la mise en place d'une comptabilité par activités (Norris, 2002), quoique affirmée comme novatrice, renvoie aux travaux antérieurs présentant les outils comptables comme socialement situés. En affirmant qu'il n'existe aucune littérature pertinente sur le sujet, l'auteur méconnaît celle-ci et ne démontre pas en quoi consiste la lacune qu'il se propose de combler.

\subsubsection{Des questions de recherche traditionnelles}

Quatre articles affichent explicitement une question déjà souvent traitée dans la recherche en contrôle. En étudiant de quoi les managers rendent compte lorsqu'ils pratiquent l'accountability, Ahrens (1996) traite de la question traditionnelle sur ce sujet (Roberts et Scapens, 1985, Roberts, 
1991, 1996). De la même manière, la question de l'impact de l'environnement culturel des acteurs sur le design et la pratique des modes de contrôle dans l'entreprise (Efferin et Hopper, 2007) est traditionnelle dans les études culturelles (Harrison et McKinnon, 1999).

Deux de ces quatre articles présentent une question de recherche traditionnelle tout en s'inscrivant explicitement dans un cadre de pensée déjà connu. Dans son étude sur le contrôle par objectifs des services publics, Modell (2003) revendique une affiliation au courant de la Nouvelle Gestion Publique (ou New Public Management, NPM) en adoptant le postulat que la performance du secteur public doit être mesurée et que les instruments comptables peuvent y contribuer. Pour sa part, Goddard (2004) traite de la manière dont les budgets sont utilisés dans la reddition de comptes du secteur public, problématique toujours en lien avec le NPM. L'adoption a priori du cadre de pensée de l'habitus bourdieusien lui permet de s'affranchir des cadres théoriques traditionnels en sciences de gestion tout en traitant de leurs questions traditionnelles.

Au final, si la majorité des articles étudiés semble faire un usage de la grounded theory dans un cadre novateur ou pour revisiter différemment des problèmes déjà bien étudiés, les méthodologies employées font état d'une très grande diversité.

\subsection{Les méthodologies adoptées dans les recherches menées}

Nous présentons dans cette section les méthodologies utilisées. Nous verrons ultérieurement si la grounded theory a permis, dans ces études, de produire de la connaissance en terme de méthodologie. Les méthodologies adoptées sont essentiellement de deux ordres et généralement alternatives. Nous avons relevé une domination des entretiens (six articles) sur les méthodes ethnométhodologiques (deux articles). Seules trois études proposent une combinaison de méthodes. 
2.3.1. Le recours à une démarche ethnométhodologique

Deux articles adoptent une démarche ethno-méthodologique (Ahrens, 1996, Wickramasinghe, et al., 2004) conformément aux propositions de Glaser et Strauss (1967).

Ahrens (1996) décrit comment il a observé deux managers britannique et allemand au travail et les a ensuite interrogés sur leurs pratiques afin de donner un contenu analytique à la description. La partie méthodologique de l'article de Wickramasinghe, Hopper et Rathnasiri (2004) montre comment, pendant toute la durée de la collecte de données, les auteurs ont été associés aux acteurs dans leurs pratiques quotidiennes. Dans un premier temps, ils ont participé à des réunions de travail au niveau de la direction de l'entreprise étudiée. Au fil des semaines, ils ont pu accéder aux niveaux les plus opérationnels, enregistrant des écritures avec les comptables et donnant avec les contremaîtres des instructions à des ouvriers. La démarche de recherche était fondée sur l'expérience et la subjectivité des chercheurs. Là aussi, des notes ont été prises et des entretiens post-opératoires conduits auprès des personnes concernées.

Dans les deux cas, les auteurs choisissent de rapporter des anecdotes tirées de leur journal de bord. Dans ces deux descriptions, les auteurs ont opté pour une théorisation fondée sur une métaphore. Chez Ahrens, les enjeux de l'accountability sont associés aux réactions de deux managers face à un toit branlant. Chez Wickramasinghe et $a l$, la gestion des coûts est associée à une forme de grève perlée dans une entreprise nippone implantée au Sri Lanka.

\subsubsection{Les études par entretiens}


Six articles optent pour une méthodologie par entretiens (Lightbody, 2000, Norris, 2002, Goddard, 2004, Goddard et Assad, 2006, Solomon et Solomon, 2006, Efferin et Hopper, 2007)

Lorsque la pratique de la grounded theory consiste seulement en la conduite d'entretiens, ceux-ci sont généralement enregistrés et retranscrits (Lightbody, 2000 ; Norris, 2002 ; Goddard, 2004 ; Goddard et Assad, 2006 ; Solomon et Solomon, 2006). Lorsque l'enregistrement n'est pas permis, les réponses aux questions font l'objet d'une première synthèse avant d'être complétées par d'autres entretiens (Efferin et Hopper, 2007). Dans tous les cas, l'analyse consiste en un codage des données textuelles collectées. Toutes les recherches ayant procédé ainsi font état du mode opératoire dans la partie méthodologique. Deux articles proposent des développements didactiques quant à l'utilité du codage (Norris, 2002) ou quant aux formes que celui-ci peut revêtir (Goddard et Assad, 2004).

\subsubsection{Les méthodes combinées}

Trois articles présentent une combinaison de méthodes. Dans deux cas (Parker, 2001, 2002), la recherche combine observation participante et entretiens. Se fondant en grande partie sur son expérience de membre de l'église étudiée, l'auteur a mené à titre exploratoire une étude ethnographique. De celle-ci ont pu émerger des catégories lui permettant de construire une grille d'entretiens semi-directifs. Ceux-ci ont pu alors être conduits auprès des dirigeants de la congrégation. C'est finalement l'analyse de ces entretiens qui a mis au jour les concepts guidant le développement d'une théorie de la planification et du processus budgétaire dans les organisations religieuses et les organisations à but non-lucratif.

Dans un cas, (Modell, 2003), la recherche combine entretiens et données secondaires issues de comptes-rendus de réunions, de rapports, de coupures de presse. Le codage de toutes ces données 
adopte le même protocole, suivant le même schéma d'interprétation. Une telle triangulation des méthodes et des données devait permettre de neutraliser certains des biais des personnes interrogées et d'avoir une vision globale du phénomène étudié.

\subsection{Les apports de la grounded theory aux articles de notre échantillon}

Les articles étudiés produisent essentiellement des connaissances théoriques et empiriques. Les apports méthodologiques sont nettement moindres.

\subsubsection{Des connaissances théoriques plus souvent novatrices que confirmatoires}

Tous les articles sélectionnés présentent un apport théorique. Deux produisent une théorie formelle aboutie, tandis que quatre autres produisent une théorie substantive en devenir. En somme, six articles sur ont un apport théorique significatif, alors que cinq autres confirment des théories déjà existantes.

\subsubsection{Des théories formelles abouties}

Une théorie formelle apparaît dans les conclusions d'Ahrens (1996). Alors que la plupart des travaux antérieurs assimilaient accountability et présentation d'états financiers, Ahrens observe qu'elle a deux dimensions, l'une financière et l'autre opérationnelle. Les managers doivent rendre compte dans des états financiers de l'utilisation de l'argent qui leur est confié. Mais ils doivent aussi légitimer par voie discursive la manière dont le projet organisationnel est conduit. Ces deux dimensions ne sont pas alternatives, mais sont complémentaires, leur prégnance variant selon les

priorités des managers. Depuis, la plupart des travaux sur l'accountability se réfèrent aux 
conclusions d'Ahrens.

Wickramasinghe et al. (2004) proposent également une théorie formelle : les modes de contrôle traditionnels sont inopérants dans les pays en développement du fait de facteurs culturels, économiques et politiques. A condition d'intégrer les facteurs culturels, économiques et politiques des pays en développement, ils peuvent avoir une vertu émancipatrice. En offrant une lecture systématique du fonctionnement de l'organisation et du pays, ces outils adaptés peuvent contribuer à la démocratisation du régime et au développement économique. Depuis, cette théorie a servi de point de référence à la plupart des travaux sur la comptabilité dans les pays en développement.

\subsubsection{Des théories substantives en devenir}

Cinq articles présentent des théories substantives. En soulignant le fossé entre la mission sacrée de l'église et le caractère séculier des comportements de protection des ressources, Lightbody (2000) enrichit la théorie du fossé sacré-séculier telle que découverte par Laughlin (1988) et Booth (1993). Selon McPhail, Gorringe et Gray (2004, 2005), cet enrichissement constitue un premier pas dans la découverte d'une théorie formelle de la comptabilité et du contrôle dans les organisations religieuses. En cela, Lightbody a offert des concepts ouvrant sur une théorie substantive enrichie à son tour par des travaux ultérieurs, dont ceux de Parker (2002).

Goddard et Assad (2006) notent que la comptabilité et le contrôle permettent de gérer la légitimité du management dans une ONG. Alors que de telles conclusions avaient déjà été tirées pour des entreprises privées (Roberts, 1991, 1996), Goddard et Assad envisagent que des travaux ultérieurs puissent enrichir et approfondir les leurs. Ainsi, ils posent les jalons pour de futurs développements sur la place de la comptabilité dans les organisations à but non-lucratif. Bien que cette contribution 
à la connaissance soit très récente, quelques travaux sur le même sujet commencent à se référer à ces conclusions (Unerman et O'Dwyer, 2008).

Trois articles présentent des théories substantives trop récentes pour avoir fait l'objet de références. Parker (2001) conclue que dans une organisation religieuse, la planification et l'instrumentation de gestion sont introduits de manière réactive et sont expliquées par quatre facteurs : la culture et le repli communautaires, la pression sur les ressources, la bureaucratie consultative et l'intérêt pour l'information comptable. A ce jour, seule une publication a porté sur des préoccupations connexes et a fait référence à la théorie développée (Kreander, McPhail et Molyneaux, 2004, 2005). Solomon et Solomon (2006) concluent que les informations éthiques, sociales et environnementales privées, c'est-à-dire adressées aux investisseurs de référence, contribuent à alimenter les informations publiques des sociétés. Efferin et Hopper (2007) concluent que dans une organisation multiethnique, les cultures sont complémentaires pour donner du contenu aux systèmes de contrôle, ceux-ci étant alors fondés sur celles-là. Dans une plus grande mesure que celle de Parker, des deux recherches sont trop récentes pour que leur portée puisse être appréciée. Nous n'avons relevé qu’une seule référence à ces deux articles (Gurd, 2008).

\subsubsection{Des théories confirmatoires de théories existantes}

Sur les quatre articles offrant des théories confirmatoires de théories existantes, deux n'apportent aucune nouveauté théorique, rappelant simplement que leur pertinence demeure d'actualité. Deux autres confirment et enrichissent des théories existantes de nouveaux points de vue.

Norris (2002) observe que la mise en place d'une comptabilité par activités est motivée par le besoin qu'expriment les managers et par la confiance qu'ils ont a priori dans l'outil, confirmant ainsi les 
conclusions de la littérature relative à la confiance et aux outils de gestion. Modell (2003) parvient à la conclusion que la direction par objectifs dans le secteur public se traduit par du loose coupling et permet de fixer des orientations. En cela, il valide les conclusions des travaux antérieurs du New Public Management.

En concluant que l'accountability ne peut être comprise qu'à l'aune des perceptions qu'en ont les individus, Goddard (2004) confirme les conclusions antérieures selon lesquelles elle résulte des interactions de différents acteurs au sein de l'organisation. Comme les recherches antérieures, Goddard observe qu'elle s'inscrit dans un schéma de demande de légitimation des comportements dans un contexte de domination et de recherche de sens pour l'action publique. Non seulement la grounded theory lui permet de confirmer une théorie de l'accountability bien connue. Mais en plus, son inscription dans le paradigme bourdieusien de l'habitus vient confirmer l'acuité des trois dimensions la fondant (signification, domination, légitimation).

La recherche de Parker (2002) est la seule de laquelle émergent des concepts explicitement présents dans des travaux antérieurs. Le codage des entretiens réalisés a mis en évidence les termes «sacré», « séculier», «profane », «conflits » qui apparaissent à plusieurs reprises dans l'article. Dans la discussion finale, l'auteur renvoie ces concepts aux travaux qui les ont vus émerger les premiers (Laughlin, 1988 ; Booth, 1993). Parker et Roffey (1997) estiment que la redécouverte d'une théorie par la grounded theory, parce que non intentionnelle, vient renforcer sa légitimité auprès de la communauté scientifique. Ce qui compte alors est la redécouverte par une voie alternative et ainsi la convergence de modes de recherche vers une même théorie.

2.4.2. Une description empirique riche ouvrant sur de nouveaux objets 
Six articles (Lightbody, 2000 ; Parker, 2001, 2002 ; Goddard, 2004 ; Wickramasinghe et al., 2004 ;

Efferin et Hopper, 2007) s'attachent à décrire minutieusement des terrains encore peu ou mal connus des chercheurs en comptabilité, alors que dans les cinq autres la description d'un objet déjà bien documenté reste relativement évasive.

Lightbody (2000) et Parker (2001, 2002), dans l'introduction de leurs articles, précisent qu'un enjeu important de leurs recherches est de porter à la connaissance de la communauté scientifique le fonctionnement et les modes de contrôle d'une organisation religieuse. Ainsi, la description est très exhaustive et précise, faisant état de nombreuses citations de personnes interrogées. Ce faisant, les auteurs montrent les enjeux quotidiens de la comptabilité, de la planification stratégique et du processus budgétaire dans une organisation religieuse.

Wickramasinghe, Hopper et Rathnasiri (2004) étudient les modes de contrôle et de travail dans une entreprise énergétique sri-lankaise. La narration détaillée faite du terrain de recherche permet au lecteur de découvrir l'organisation et ses modes de contrôle comme s'il y était. De la même manière, Efferin et Hopper (2007) décrivent avec une très grande précision les traits des ethnies indonésiennes et chinoises selon que leurs représentants sont minoritaires ou majoritaires dans l'organisation. Pour cela, ils se fondent aussi bien sur de nombreux extraits de verbatim que sur d'abondantes données secondaires relatives à ces deux groupes ethniques.

\subsubsection{Des débats méthodologiques stimulants}

Seuls trois articles (Modell, 2003 ; Ahrens, 1996 ; Efferin et Hopper, 2007) affichent ouvertement une contribution à la connaissance par des apports méthodologiques. 
La partie méthodologique de l'article de Modell (2003) fournit une présentation détaillée des différentes méthodes qui seront combinées et de la manière dont elles seront croisées pour extraire des points de convergence théorique. Le croisement des données et des méthodes conduit à un recoupement des critères de validité de la recherche qualitative et apparaît comme une démarche novatrice permettant de mieux trianguler les données.

Pour leur part, Ahrens (1996) et Efferin et Hopper (2007) proposent une méthode pour appréhender les études culturelles et les comparaisons. Selon eux, l'étude des cultures ne constitue pas une fin en soi, mais doit permettre d'identifier des traits communs aux outils de gestion observés.

L'apport méthodologique de l'article d'Ahrens tient au fait que les cultures ne sont pas étudiées en tant que telles. Elles sont considérées comme un élément de contexte dans lequel est pratiquée la gestion. La compréhension des spécificités du contexte doit contribuer à produire de la connaissance sur l'objet comptable étudié. Selon Ahrens, ce ne sont pas les conclusions anthropologiques de chercheurs en comptabilité qui font progresser la connaissance comptable. D’un point de vue méthodologique, Ahrens resitue les études culturelles dans le cycle de production de connaissance comptable.

Tout comme Ahrens, Efferin et Hopper (2007) considèrent les cultures comme un élément de contexte de la recherche. Néanmoins, ils divergent sur la place des cultures dans le processus de production de connaissance comptable. Efferin et Hopper considèrent que des conclusions théoriques sur le contexte culturel enrichit la compréhension d'un objet comptable. Au-delà des cultures, Efferin \& Hopper encouragent le chercheur à mener des recherches transdisciplinaires afin de bien cerner les enjeux d'un contexte spécifique. A la différence d'Ahrens, ils considèrent que la production de connaissances non comptables peut s'inscrire dans le cycle de production de 
connaissances en comptabilité.

Il ressort de ce panorama que les apports méthodologiques effectifs des recherches étudiées sont assez pauvres. Ces recherches mettent en évidence au travers d'études de cas des débats épistémologiques et méthodologiques traditionnellement abordés dans des articles exclusivement épistémologiques ou méthodologiques. Un apport peut consister alors en l'opérationnalisation d'un positionnement épistémologique et méthodologique. C'est de cette logique que relève le long développement et l'annexe sur la grounded theory de l'article de Efferin et Hopper (2007).

\subsection{Les critères de scientificité retenus}

Nous pouvons noter qu'il existe autant de manières de défendre la scientificité de la connaissance produite que d'articles, ceci étant en grande partie dû à la combinaison de critères.

\subsubsection{Le recours au codage de données textuelles}

Les auteurs ayant recours à des entretiens enregistrés puis retranscrits disent tous les avoir codés, cette procédure d'analyse revêtant un caractère scientifique et vérifiable. Tous les auteurs ne font pas état de leurs techniques de codage et de leurs résultats de la même manière. Norris (2002) présente dans le corps du texte les catégories qu'il a pu obtenir en codant et en annexes des extractions du logiciel NUD*IST qu'il a utilisé. Le lecteur peut également trouver un extrait d'entretien ainsi que l'analyse sémantique permise par le logiciel et toutes les étapes de son codage jusqu'à la sélection du verbatim cité dans le corps du texte. Le chercheur a ainsi laissé des traces vérifiables de son analyse, conformément à ce que recommandent Strauss et Corbin (1998). 
D’autres travaux proposent une vérifiabilité partielle (Goddard et Assad, 2005 ; Solomon, 2006). Solomon et Solomon (2006) ont choisi de présenter dans le corps du texte les catégories auxquelles les a conduits leur codage et tout le processus d'analyse. En revanche, le lecteur n'a aucune trace des extractions du logiciel utilisé. Il ne peut apprécier la scientificité de la recherche qu'à l'aune des clés de compréhension systématiques fournies. Goddard et Assad (2005) ont préféré donner aux extraits du logiciel une forme plus facilement lisible en les retraitant. Le lecteur ne dispose que des catégories et des thèmes ressortant dans le discours des acteurs. Nulle part n'apparaissent le cheminement complet du codage ni les conclusions telles que le logiciel les a effectivement présentées. De la même manière, Parker (2001) expose les quatre facteurs découverts dans l'étude ethnographique comme les catégories de sa matrice de codage. Les autres travaux fondés sur des entretiens ne présentent pas au lecteur de voies pour apprécier la traçabilité du discours et ainsi la validité de la recherche (Lightbody, 2000 ; Parker, 2002 ; Goddard, 2004 ; Efferin et Hopper, 2007). Lorsque le codage n'est pas présenté, la scientificité repose plutôt sur la plausibilité et la cohérence du discours.

\subsubsection{La plausibilité et la cohérence du discours}

La plausibilité tient au caractère exhaustif et non superflu des descriptions fournies. Les cinq recherches optant pour ce critère de validité présentent les objets de telle sorte que le lecteur puisse se représenter la situation, en anticiper les enjeux et se sentir concerné face aux situations de gestion qu'il rencontre (Ahrens, 1996 ; Lightbody, 2000 ; Parker, 2001 ; Modell, 2003 ; Wickramasinghe et al., 2004).

Lightbody (2000), Parker (2001), Modell (2003) et Goddard (2004) soulignent les points de convergence des différents discours. De là, ils tentent de reconstituer un métadiscours 
organisationnel dans lequel les discours individuels pourraient trouver leur place. La plausibilité tient alors au fait de montrer la cohésion, la convergence et la cohérence des propos tenus par les différents acteurs dans l'organisation pour que le lecteur puisse se la représenter. Parker révèle la cohésion des managers autour de la nécessité de disposer d'outils de gestion et la cohésion des ministres du culte autour du rejet de ceux-ci. Modell montre la cohérence du discours des dirigeants politiques nationaux et locaux face à la mesure des performances, et celle des propos des dirigeants des universités interrogés pour montrer leur perplexité à l'égard d'une direction par objectif et enfin du corps enseignant et étudiant considérant impossible de mesurer des performances pédagogiques et scientifiques. Goddard, en s'intéressant seulement à des élus locaux, montre la convergence des positions individuelles face à l'élaboration des budgets: ils perçoivent le processus budgétaire et l'utilisation des budgets comme autant d'outils de transparence à l'attention du public. La plausibilité des articles d'Ahrens et de Wickramasinghe et al. se trouve dans la simplicité et le caractère quotidien des anecdotes rapportées. Ahrens rapporte les réactions managériales face à un toit branlant. Wickramasinghe et al. présentent la condition des gens au travail et diverses altercations entre ouvriers. La plausibilité du discours n'est pas une donnée, elle est appréciée subjectivement par le chercheur lui-même et son lecteur. Sa quête implique la réflexivité des acteurs, y compris le chercheur, à l'égard du texte.

\subsubsection{La réflexivité des acteurs et du chercheur vis-à-vis du texte}

Les chercheurs ont parfois soumis aux personnes qu'ils ont rencontrées leurs observations ou le fruit des entretiens qu'ils ont pu conduire. Leurs commentaires ont été enregistrés afin d'ajuster l'écriture. Autant d'allers et retours vers le terrain permettent in fine de disposer d'un discours fidèle à la réalité des perceptions des acteurs organisationnels. 
Dans quatre cas (Ahrens, 1996 ; Lightbody, 2000 ; Wickramasinghe, Hopper et Rathnasiri, 2004 ; Efferin et Hopper, 2007), les chercheurs associent méthodes ethnographiques et réflexivité des acteurs vis-à-vis du texte, conformément aux préconisations de Glaser et Strauss (1967). Dans leur partie méthodologique, les quatre articles spécifient que les observations émises et les analyses tirées ont été soumises systématiquement à l'appréciation des personnes observées. Lorsque le chercheur se fonde sur une démarche ethnométhodologique, il soumet les anecdotes observées et l'interprétation qu'il en fait aux acteurs organisationnels pour confirmation. Ahrens a soumis ses observations aux managers qui se sont parfaitement reconnus dans leur situation professionnelle. Lightbody a fait de même auprès des comptables et des ministres du culte de l'Eglise d'Australie. Wickramasinghe et al. ont pour leur part rapporté les anecdotes aux personnes avec lesquelles ils ont travaillé pour s'assurer qu'elles confirmaient bien retrouver leurs réactions et comportements d'alors.

Efferin et Hopper (2007) indiquent dans les annexes de leur article avoir eu recours à une double réflexivité (la leur et celle des personnes observées) du fait de l'interdiction qui leur était faite d'enregistrer les entretiens. Afin de démontrer la vérifiabilité de leur recherche, ils déclarent avoir interrogé à plusieurs reprises les mêmes personnes en leur posant les mêmes questions et en soumettant à leur approbation les réponses antérieures qu'ils avaient pu produire. La capacité réflexive des acteurs a été sollicitée à plusieurs occasions afin de révéler la cohérence et partant la plausibilité des discours.

\section{Conclusion}

Notre article s'interroge sur les conditions dans lesquelles la grounded theory peut contribuer à la connaissance scientifique. L'analyse des travaux de recherche en contrôle de gestion a permis 
d'identifier des voies plurielles vers la production de connaissances à l'aide d'une démarche de grounded theory. Tous les travaux étudiés avaient en commun de reposer sur des techniques et procédures systématiques dans le cadre de recherches interprétatives de type abductif. Toutes ont en commun d'opérationnaliser les suggestions évasives de Glaser et Strauss et de Strauss et Corbin. Le fait que notre échantillon présente plusieurs articles du même auteur (Parker, 2001, 2002 ; Efferin et Hopper, 2007 ; Wickramasinghe, Hopper et Rathnasiri, 2004) illustre bien le fait que la grounded theory est un mode de recherche à part entière. Strauss et Corbin (1998), Parker et Roffey (1997), Locke (2000) et Gurd (2008) insistent sur le fait que c'est un mode de pensée cohérent qui ne laisse pas le chercheur indemne après qu'il l'a pratiquée une fois.

La pluralité d'utilisations de la grounded theory tient à la pluralité des possibles à chaque étape de la recherche. En effet, pour chacune des catégories guidant notre analyse nous avons pu identifier plusieurs sous-catégories, chacune offrant une particularité. Si chacune regroupe un certain nombre de travaux, le cheminement de la nature de la question de recherche à la contribution à la connaissance, en passant par la méthodologie employée et les critères de validité retenus reste la spécificité du terrain et du chercheur. En effet, nous n'avons pas identifié deux articles entrant dans les mêmes catégories tout au long du processus de recherche. Nous avons identifié onze manières de pratiquer la grounded theory.

Loin d'être une approche alternative au modèle hypothético-déductif traditionnel, dans les faits, la grounded theory évolue à ses côtés de manière complémentaire. Nous avons constaté que souvent les découvertes de la grounded theory peuvent achever de valider des théories issues d'autres démarches de recherche. Symétriquement, lorsque des théories nouvelles sont développées, elles peuvent ensuite servir d'hypothèses que des recherches postérieures testent avec une autre approche. De futures recherches pourront partir de ces conclusions pour proposer d'étudier en 
profondeur l'articulation de la grounded theory et de démarches hypothético-déductives dans le cycle de production de théories nouvelles.

La principale implication pratique de cette recherche est de fournir aux chercheurs francophones en comptabilité désireux de s'initier à la pratique de la grounded theory un éclairage sur les enjeux qu'ils pourront être conduits à traiter. Une deuxième implication pratique est propre à rassurer la communauté scientifique francophone quant à l'intérêt et la place de ce type d'approche dans les paradigmes de recherche en contrôle de gestion.

\section{Bibliographie:}

Ahrens, T. (1996). Styles of accountability. Accounting, Organizations and Society 21 (2-3): 139173.

Booth, P. (1993). Accounting in churches: A research framework and agenda. Accounting, Auditing \& Accountability Journal 6 (4): 37-67.

Chambost, I. (2007). Contribution à l'analyse de la formation du jugement des analystes financiers sell-side. Paris: Conservatoire National des Arts et Métiers.

Charreire, S. et Huault, I. (2002). Le constructivisme dans la pratique de recherche : Une évaluation à partir de seize thèses de doctorat. Finance Contrôle Stratégie 4: 31-55.

David, A. (2000). Logique, épistémologie et méthodologie en sciences de gestion: Trois hypothèses revisitées In Les nouvelles fondations des sciences de gestion (Eds, A. David, A. Hatchuel and R. Laufer). Paris: FNEGE-Vuibert, 83-109.

Durocher, S., Fortin, A. et Cote, L. (2004). Pouvoir et normalisation : La perception des utilisateurs canadiens. Comptabilité Contrôle Audit ${ }^{\circ}$ thématique (juin): 193-212.

Efferin, S. et Hopper, T. (2007). Management control, culture and ethnicity in a chinese indonesian company. Accounting, Organizations and Society 32 (3): 223-262.

Glaser, B. et Strauss, A. (1967). The discovery of grounded theory: Strategies of qualitative research. London: Wiedenfeld and Nicholson.

Goddard, A. (2004). Budgetary practices and accountability habitus: A grounded theory. Accounting, Auditing \& Accountability Journal 17 (4): 543 - 577.

Goddard, A. et Assad, M. J. (2006). Accounting and navigating legitimacy in tanzanian ngos. Accounting, Auditing \& Accountability Journal 19 (3): 377 - 404.

Gurd, B. (2008). Remaining consistent with methods? An analysis of grounded theory research in accounting. Qualitative Research in Accounting \& Management 5 (2): 122-138.

Harrison, G. L. et McKinnon, J. L. (1999). Cross-cultural research in management control systems design: A review of the current state. Accounting, Organizations and Society 24 (5-6): 483-506.

Howard, T. et Stout, D. (2006). Reasons accounting case/instructional resource papers are rejected for publication. Journal of Accounting Education 24: 1-15.

Laughlin, R. (1995). Empirical research in accounting: Alternative approaches and a case for "Middle-range" Thinking. Accounting, Auditing \& Accountability Journal 8 (1): 63 - 87.

Lightbody, M. (2000). Storing and shielding: Financial management behaviour in a church 
organization. Accounting, Auditing \& Accountability Journal 13 (2): 156 - 174.

McPhail, K., Gorringe, T. et Gray, R. (2004). Accounting and theology, an introduction: Initiating a dialogue between immediacy and eternity. Accounting, Auditing \& Accountability Journal 17 (3): $320-326$.

McPhail, K., Gorringe, T. et Gray, R. (2005). Crossing the great divide: Critiquing the sacred secular dichotomy in accounting research. Accounting, Auditing \& Accountability Journal 18 (2): $185-188$.

Modell, S. (2003). Goals versus institutions: The development of performance measurement in the swedish university sector. Management Accounting Research 14 (4): 333-359.

Nobre, T. (2001). Management hospitalier : Du contrôle externe au pilotage, apport et adaptabilité du tableau de bord prospectif. Comptabilité Contrôle Audit 7 (2): 125-146.

Norris, G. (2002). Chalk and cheese: Grounded theory case studies of the introduction and usage of activity-based information in two british banks The British Accounting Review 34: 223-255.

Parker, L. D. (2001). Reactive planning in a christian bureaucracy. Management Accounting Research 12 (3): 321-356.

Parker, L. D. (2002). Budgetary incrementalism in a christian bureaucracy. Management Accounting Research 13 (1): 71-100.

Quattrone, P. (2000). Constructivism and accounting research: Towards a trans-disciplinary perspective. Accounting, Auditing \& Accountability Journal 13 (2): 130 - 155.

Richard, C. et Reix, R. (2002). Contribution à l'analyse de la qualité du processus d'audit : Le rôle de la relation entre le directeur financier et le commissaire aux compte. Comptabilité Contrôle Audit 1 (mai): 151-174.

Roberts, J. (1991). The possibilities of accountability. Accounting, Organizations and Society 16 (4): 355-368.

Roberts, J. (1996). From discipline to dialogue: Individualizing and socialising forms of accountability. In Accountability: Power, ethos and the technologies of managing (Eds, R. Munro and J. Mouritsen). London: International Thomson Business Press, 40-61.

Roberts, J. et Scapens, R. (1985). Accounting systems and systems of accountability -understanding accounting practices in their organisational contexts. Accounting, Organizations and Society 10 (4): 443-456.

Solomon, J. et Solomon, A. (2006). Private social, ethical and environmental disclosure. Accounting, Auditing \& Accountability Journal 19: 564-591.

Strauss, A. et Corbin, J. (1990). Basics of qualitative research: Techniques and procedures for developing grounded theory. Newbury Park: Sage publishing.

Strauss, A. et Corbin, J. (1998). Basics of qualitative research: Techniques and procedures for developing grounded theory. Thousand Oaks: Sage.

Thiétart, R.-A. (1999). Méthodes de recherche en management. Paris: Dunod.

Unerman, J. et O'Dwyer, B. (2008). The paradox of greater ngo accountability : A case study of amnesty ireland. Accounting, Organizations \& Society a paraître.

Villesèque-Dubus, F. (2005). Vers une transversalisation des budgets : Un essai d'observation et d'interprétation. Comptabilité Contrôle Audit 11 (2): 127-147.

Wickramasinghe, D., Hopper, T. et Rathnasiri, C. (2004). Japanese cost management meets sri lankan politics: Disappearance and reappearance of bureaucratic management controls in a privatised utility. Accounting, Auditing \& Accountability Journal 17 (1): 85 - 120. 


\section{Annexe 1: Synthèse de la variété d'utilisation de la grounded theory}

\begin{tabular}{|c|c|c|c|c|}
\hline Article & \multicolumn{2}{|c|}{ Question de recherche } & \multicolumn{2}{|c|}{ Méthodologie } \\
\hline & Expression de la question de recherche & $\begin{array}{c}\text { Nouveauté ou préexistence de la question de } \\
\text { recherche }\end{array}$ & $\begin{array}{l}\text { Positionnement par rapport aux } \\
\text { cadres théoriques préxistants }\end{array}$ & Modes opératoires \\
\hline $\begin{array}{l}\text { Ahrens (1996), Styles of Accountability, } \\
\text { AOS }\end{array}$ & $\begin{array}{l}\text { De quoi un manager rend-il compte quand i } \\
\text { pratique l'accountability? }\end{array}$ & $\begin{array}{l}\text { Question traditionnelle } \\
\text { C'est la question traditionnelle des études sur } \\
\text { l'accountability }\end{array}$ & \begin{tabular}{|l|} 
Référence aux études de Roberts et \\
Scapens (1985) et Roberts $(1990,1991)$ \\
sur les dimensions de l'accountability
\end{tabular} & Ethno-méthodologie (Garfinkel) \\
\hline $\begin{array}{l}\text { Lightbody (2000), Storing and shielding: } \\
\text { financial management behaviour in a } \\
\text { church organization, AAAJ }\end{array}$ & $\begin{array}{l}\text { Quelle est la nature des comportements de } \\
\text { gardien des ressources et de défenseurs de la } \\
\text { mission dans une organisation religieuse? }\end{array}$ & $\begin{array}{l}\text { Question faussement nouvelle } \\
\text { Depuis les travaux de Laughlin (1988) et de Booth } \\
\text { (1993), cette question est devenue la question-type } \\
\text { des recherches sur la comptabilité des } \\
\text { congrégations religieuses (McPhail, Gorringe \& } \\
\text { Gray, 2004, 2005) }\end{array}$ & $\begin{array}{l}\text { Référence aux études Laughlin (1988) et } \\
\text { de Booth (1993) auxquels l'auteure } \\
\text { emprunte les concepts qu'elle utilise } \\
\text { pour développer sa propre théorie. }\end{array}$ & $\begin{array}{l}\text { Démarche ethnographique fondée sur } \\
\text { des entretiens semi-directifs et suivant } \\
\text { Strauss \& Corbin (1998) }\end{array}$ \\
\hline $\begin{array}{l}\text { Norris (2002), Chalk and cheese: } \\
\text { grounded theory case studies of the } \\
\text { introduction and usage of activity-based } \\
\text { information in two Irish banks, BAR }\end{array}$ & $\begin{array}{l}\text { Quels facteurs influencent la mise en place et } \\
\text { l'utilisation d'une comptabilité par activités? }\end{array}$ & $\begin{array}{l}\text { Question faussement nouvelle } \\
\text { L'auteur estime qu'il existe très peu de travaux su } \\
\text { les facteurs influençant l'utilisation de techniques } \\
\text { de contrôle, en dehors de ceux de Hopwood sur la } \\
\text { comptabilité en tant que pratique socialement } \\
\text { située. Alors qu'il semb }\end{array}$ & $\begin{array}{l}\text { Référence aux travaux relatifs } \\
\text { l'information comptable }\end{array}$ & $\begin{array}{l}\text { Entretiens semi-directifs suivant la } \\
\text { méthodologie de Strauss \& Corbin } \\
(1990)\end{array}$ \\
\hline $\begin{array}{l}\text { Parker (2001), Reactive planning in a } \\
\text { Christian bureaucracy, MAR }\end{array}$ & $\begin{array}{l}\text { Quels sont la nature et les facteurs } \\
\text { conditionnant la planification dans une } \\
\text { organisation religieuse? }\end{array}$ & $\begin{array}{l}\text { Question nouvelle } \\
\text { Compte tenu du faible nombre de recherches } \\
\text { antérieures sur la comptablité des congrégatinos } \\
\text { religieuses, la question de recherche est novatrice. }\end{array}$ & $\begin{array}{l}\text { Les travaux antérieurs sur un fossé entre } \\
\text { le sacré et le séculier (Laughlin 1988 } \\
\text { Booth, 1993; Lightbody, 2000) sont } \\
\text { présentés et discutés en fin d'article. }\end{array}$ & $\begin{array}{l}\text { Démarche ethnographique fondée sur } \\
\text { de l'observation participante complétée } \\
\text { par des entretiens semi-directifs. }\end{array}$ \\
\hline $\begin{array}{l}\text { Parker (2002), Budgetary incrementalism } \\
\text { in a Christian bureaucracy, MAR }\end{array}$ & $\begin{array}{l}\text { Par quels moyens les processus de planification } \\
\text { sont reliés les uns avec les autres e } \\
\text { interagissent avec les processus budgétaires et } \\
\text { le processus de contrôle d'une organisation } \\
\text { religieuse? En quoi cela diffère-t-il d'une } \\
\text { entreprise privée? }\end{array}$ & $\begin{array}{l}\text { Question nouvelle } \\
\text { Cet article est un des premiers de la recherche } \\
\text { comptable à proposer d'identifier les spécificités } \\
\text { des organisations à but non lucratif. }\end{array}$ & $\begin{array}{l}\text { Référence aux travaux antérieurs relatifs } \\
\text { à la planification budgétaire et au } \\
\text { contrôle. Aucune référence aux travaux } \\
\text { antérieurs sur la comptablité et le } \\
\text { contrôle des organisations à but non } \\
\text { lucratif. }\end{array}$ & $\begin{array}{l}\text { Démarche ethnographique fondée sur } \\
\text { de l'observation participante complétée } \\
\text { par des entretiens semi-directifs. }\end{array}$ \\
\hline
\end{tabular}




\begin{tabular}{|c|c|c|c|c|}
\hline $\begin{array}{l}\text { Modell (2003), Goals versus institutions: } \\
\text { the development of performance } \\
\text { measurement in the Swedish university } \\
\text { sector, MAR }\end{array}$ & $\begin{array}{l}\text { Le secteur public peut-il être dirigé par } \\
\text { objectifs? }\end{array}$ & $\begin{array}{l}\text { Question traditionnelle } \\
\text { C'est une des questions fondatrices du mouveme } \\
\text { de la Nouvelle Gestion Publique. }\end{array}$ & $\begin{array}{l}\text { Référence aux travaux antérieurs sur la } \\
\text { Nouvelle Gestion Publique. }\end{array}$ & $\begin{array}{l}\text { Entretiens semi-directifs triangulés } \\
\text { avec d'autres sources de données. }\end{array}$ \\
\hline \begin{tabular}{|l|} 
Wickramasinghe, Hopper \& Rathnasiri \\
(2004), Japanese cost management meets \\
Sri Lankan politics - Disappearance and \\
reappearance of bureaucratic managemer \\
controls in a privatised utility, AAAJ
\end{tabular} & $\begin{array}{l}\text { Comment les modes de contrôle de gestion } \\
\text { sont liés, de manière imprévisible, dans les pa } \\
\text { en développement à des facteurs culturels } \\
\text { économiques et politiques? }\end{array}$ & $\begin{array}{l}\text { Question nouvelle } \\
\text { Ce sont les pays en développementqui constituen } \\
\text { la nouveauté }\end{array}$ & $\begin{array}{l}\text { L'article adoptant la méthodologid } \\
\text { préconisée par Glaser et Strauss, le text } \\
\text { ne présente pas de revue de littérature } \\
\text { Les cocnepts employés sont restitués in } \\
\text { vivo à leurs auteurs. }\end{array}$ & Ethno-méthodologie \\
\hline $\begin{array}{l}\text { Goddard (2004), Budgetary practices an } \\
\text { accountability habitus - a grounded theor } \\
\text { AAAJ }\end{array}$ & $\begin{array}{l}\text { Comment les budgets sont-ils utilisés pout } \\
\text { rendre compte dans des collectivités locales? }\end{array}$ & $\begin{array}{l}\text { Question traditionnelle } \\
\text { Cette question est largement traitée dans les } \\
\text { recherches sur la nouvelle gestion publique }\end{array}$ & \begin{tabular}{|l|} 
Enrichissement du cadre théorique de \\
l'habitus de Bourdieu adoptéa priori.
\end{tabular} & Entretiens semi-directifs \\
\hline $\begin{array}{l}\text { Solomon \& Solomon (2006), Private } \\
\text { social, ethical and environmental } \\
\text { disclosure, AAAJ }\end{array}$ & $\begin{array}{l}\text { Dans quelle mesure les annonces sociales } \\
\text { éthiques et environnementales sont intégrées } \\
\text { dans l'investissement socialement responsable }\end{array}$ & $\begin{array}{l}\text { Question nouvelle } \\
\text { La nouveauté de la question réside dans la dialogic } \\
\text { entre demande dinformations privées et } \\
\text { production d'informations publiques. }\end{array}$ & $\begin{array}{l}\text { Revue de littérature visant à montrer le } \\
\text { faible nombre de recherches sur le suje }\end{array}$ & $\begin{array}{l}\text { Entretiens semi-directifs suivant la } \\
\text { méthodologie de Strauss \& Corbin } \\
(1990)\end{array}$ \\
\hline $\begin{array}{l}\text { Goddard \& Hassad (2006), Accounting } \\
\text { and navigating legitimacy in Tanzanian } \\
\text { NGOs, AAAJ }\end{array}$ & $\begin{array}{l}\text { Quels facteurs sous-tendent les procédures et } \\
\text { le reporting comptables dans une ONG? }\end{array}$ & $\begin{array}{l}\text { Question nouvelle } \\
\text { Compte tenu du faible nombre de recherche: } \\
\text { antérieures sur la comptablité des organisations à } \\
\text { but non lucratif, la question présente un carctér } \\
\text { novateur pour la recherche comptable. }\end{array}$ & $\begin{array}{l}\text { Présentation des travaux existants sur le } \\
\text { management et sur l'accountability dans } \\
\text { les organisations à but non lucratif. }\end{array}$ & $\begin{array}{l}\text { Entretiens semi-directifs suivant la } \\
\text { méthodologie de Strauss \& Corbir } \\
(1990)\end{array}$ \\
\hline $\begin{array}{l}\text { Efferin \& Hopper (2007), Management } \\
\text { control, culture and ethnicity in a Chines } \\
\text { Indonesian company, AOS }\end{array}$ & $\begin{array}{l}\text { Comment l'environnement culturel des } \\
\text { hommes d'affaires indonésiens d'origine } \\
\text { chinoise influence le design et le } \\
\text { fonctionnement des systèmes de contrôle de } \\
\text { leur entreprise? }\end{array}$ & $\begin{array}{l}\text { Question traditionnelle } \\
\text { Toutes les études culturelles en contrôle se posen } \\
\text { la même question }\end{array}$ & $\begin{array}{l}\text { Référence aux travaux de Harrison et } \\
\text { Mc Kinnon (1999). Les auteurs les } \\
\text { déconstruisent afin de mettre à jour les } \\
\text { postulats de départ et leur influence su1 } \\
\text { la recherche. }\end{array}$ & $\begin{array}{l}\text { Entretiens semi-directifs traduits et } \\
\text { retranscrits, entretiens post-opératoire }\end{array}$ \\
\hline
\end{tabular}




\section{Annexe 2. Synthèse de la variété des connaissances produites et validées}

\begin{tabular}{|c|c|c|c|c|}
\hline \multirow[t]{2}{*}{ Article } & \multicolumn{3}{|c|}{ Apports } & \multirow[t]{2}{*}{ Scientificit $^{2}$} \\
\hline & Thºriques & Empiriques & Mthodologiques & \\
\hline Ahrens (1996) & $\begin{array}{l}\text { Th`orie formelle } \\
\text { La reddition des comptes comporte une dimension financi, re et op`rationnelle }\end{array}$ & & $\begin{array}{l}\text { Les diff`rences culturelles ne sont pas `tudi`es } \\
\text { pour elles-m } \square \text { mes. En tant qu`l`ment de } \\
\text { contexte, elles`clairent sur des pratiques et } \\
\text { outils de gestion. Une `tude culturelle n'a pas } \\
n^{`} \text { cessairement pour objet la comparaison. }\end{array}$ & $\begin{array}{l}\text { Plausibilit` et } \text { r`flexivit }^{`} \text { du } \\
\text { lecteur externe }\end{array}$ \\
\hline Lightbody (2000) & 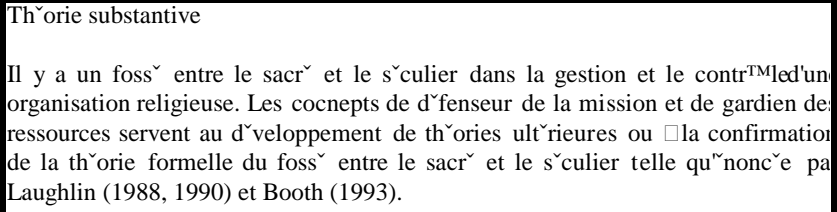 & Une congr ${ }^{2}$ gation religieuse. & & 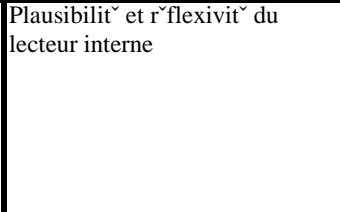 \\
\hline Norris (2002) & $\begin{array}{l}\text { Thºrie confirmatoire } \\
\text { La mise en place d'une comptabilit }{ }^{\longleftarrow} \text { par activti`s est motiv`e par le besoil } \\
\text { qu'expriment les managers et par la confiance qu'ils ont } a \text { priori dans l'outil. }\end{array}$ & & & $\begin{array}{l}\text { Codage, et extraits de NUD*IST } \\
\text { en annexes, explicitation de la } \\
\text { matrice de codage adopt`e tout } \\
\text { au long de l'article }\end{array}$ \\
\hline Parker (2001) & $\begin{array}{l}\text { Thॅorie substantive } \\
\text { Quatre facteurs influen“ant la planification strategique dans les organisations } \square \text { bu } \\
\text { non lucratif sont identifis: la pression sur les ressources, la culture e } \\
\text { l'appartenance organisationnelles, une bureaucratie consultative et la comptabilit } \\
\text { en tant que systs me de mise en conformit }{ }^{`} \text {. Ces facteurs servent de base de travai } \\
\square \text { des travaux ultrieurs sur le contr(rMledans les organisations religieuses (voi } \\
\text { McPhail, Gorringe \& Gray, 2004, 2005). }\end{array}$ & Une congr ${ }^{2}$ gation religieuse. & & Plausibiliť \\
\hline$\overline{\text { Parker (2002) }}$ & 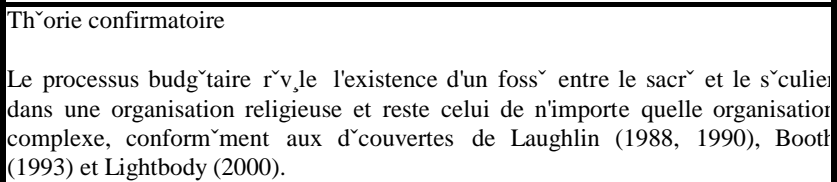 & Une congr "gation religieuse. & & Codage \\
\hline
\end{tabular}




\begin{tabular}{|c|c|c|c|c|}
\hline Modell (2003) & $\begin{array}{l}\text { Th`orie confirmatoire } \\
\text { La direction par objectifs dans le secteur public peut aider } \square \text { fixer des orientation } \\
\text { mais au final, c'est du loose coupling. Il s'agit d'une th`orie fondatrices de } 1 \\
\text { Nouvelle Gestion Publique. }\end{array}$ & & $\begin{array}{l}\text { Triangulation des donn`es et des mthodes } \\
\text { d'analyse }\end{array}$ & $\begin{array}{l}\text { Triangulation des donn`es, } \\
\text { convergences }\end{array}$ \\
\hline $\begin{array}{l}\text { Wickrmanansinghe, Hopper \& } \\
\text { Rathnasiri (2004) }\end{array}$ & 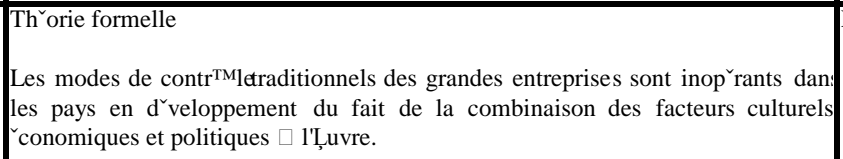 & Les pays en d`veloppement & & 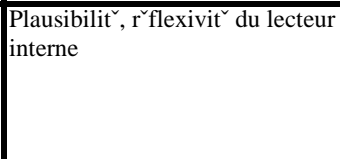 \\
\hline Goddard (2004) & 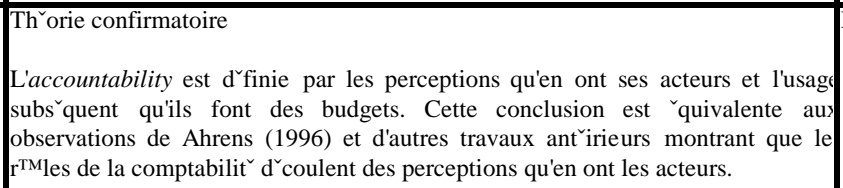 & Les collectivits locales & & Codage \\
\hline Solomon \& Solomon (2006) & $\begin{array}{l}\text { Thºrie nouvelle } \\
\text { Au-del } \square \text { des rapports de } \mathrm{d}^{\vee} \text { veloppement durable, les annonces priv`es `thiques } \\
\text { sociales et environnementales jouent un } \mathrm{r}^{\mathrm{TM}} \text { lecrucial aupr }{ }_{s} \mathrm{~s} \text { des investisseur } \\
\text { socialement responsables et s'incorporent parfaitement aux annonces financi, res } \\
\text { traditionelles. }\end{array}$ & & Sur le codage de donn es textuelles en pratique & $\begin{array}{l}\text { Codage, cat }{ }^{5} \text { gories dans le corps } \\
\text { du texte }\end{array}$ \\
\hline Goddard \& Hassad (2006) & 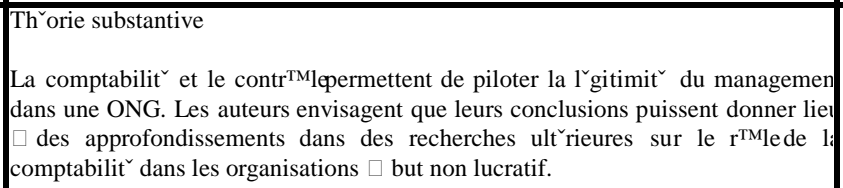 & & & Codage, cat ${ }^{\top}$ gories en annexes \\
\hline Efferin \& Hopper (2007) & \begin{tabular}{|l|} 
Thºrie substantive \\
Les facteurs culturels (ethniques) et les syst, mes de contr ${ }^{\mathrm{TM}}$ le interagissent dans le \\
design organisationnel sans que l'on soit en mesure de dire lequel influence l'autre
\end{tabular} & $\begin{array}{l}\text { Les minorits ethniques dans } \\
\text { l'organisation }\end{array}$ & 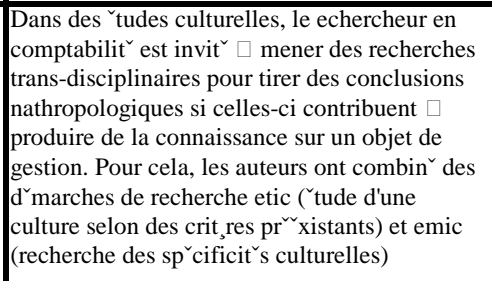 & $\overline{R^{\vee} f l e x i v i t^{2} \text { du lecteur interne }}$ \\
\hline
\end{tabular}

